\title{
Sensory Motor Polyneuropathy in a COVID 19 Patient: An Interesting Case Report
}

\section{Pushpanjali R Ojha ${ }^{1 *}$, Mohd Saif Khan ${ }^{2}$, Pradip Kumar Bhattacharya ${ }^{3}$, Aneesha Thomas ${ }^{4}$ and Kritika Raj ${ }^{5}$}

${ }^{1}$ Assistant Professor (Pathology), Trauma Center, RIMS, Ranchi, Jharkhand, India

${ }^{2}$ Associate Professor, Department of Critical Care Medicine, RIMS, Jharkhand, India

${ }^{3}$ Professor and HOD, Department of Critical Care Medicine, Trauma Center, RIMS,

Jharkhand, India

${ }^{4}$ Assistant Professor, Department of Neurology, RIMS, Ranchi, India

${ }_{5}^{5}$ Junior Resident, Department of Anaesthesia, RIMS, Ranchi, India

*Corresponding Author: Pushpanjali R Ojha, Assistant Professor (Pathology),

Trauma Center, RIMS, Ranchi, Jharkhand, India.
Received: July 08, 2021

Published: July 28, 2021

(C) All rights are reserved by Pushpanjali R

Ojha., et al.

\begin{abstract}
Background: The neurotropic effect of SARS - CoV2 virus manifests as inflammatory and demyelinating changes. The major entities reported were stroke, GBS, ADEM and encephalitis. A rare case of sensory motor polyneuropathy (SMPN) in COVID 19 was reported in our institute.

Methods: A COVID19 diseased patient without any previous neurological disorders was admitted and during the course of treatment developed neurological complications. After complete radiological and pathological evaluation he was diagnosed as a case of SMPN - a new manifestation of SARS - CoV2 infection.

Results: HRCT chest shows COVID 19 related ARDS, MRI (T2W) at D6-10 spines shows subtle patchy intramedullary high signal intensity and Nerve conduction study revealed asymmetrical sensory-motor polyneuropathy. He had leucocytosis with neutrophilia, lymphopenia, raised Neutrophil-lymphocyte ratio, D-Dimer and ferritin.

Conclusion: SMPN is relatively rare but acute manifestation of SARS-CoV2 infection which need immediate management and long term follow up to diagnose chronic complication related with it.
\end{abstract}

Keywords: Guillain - Barre Syndrome; Sensory Motor Polyneuropathy; Nerve Conduction Study

\section{Abbreviations}

RT-PCR: Reverse Transcriptase Polymerase Chain Reaction; ARDS: Acute Respiratory Distress Syndrome; HRCT: High Resolution Computed Tomography; T2W MRI: T 2 Weighted Magnetic Resonance Imaging; PT: Prothrombin Time; SGOT: Serum Glutamic Oxalo-Acetic Transaminase; SGPT - Serum Glutamate Pyruvate Transaminase; ALP: Alkaline Phosphatase; LDH: Lactate Dehydrogenase; CMAP: Compound Muscle Action Potential

\section{Introduction}

SARS-CoV2 virus show neurotropism in addition to cytotropism with cells of multiple organs. Several recent studies had reported CNS manifestations that range from encephalitis, myelitis, radiculitis, plexitis, Guillan-Barrè syndrome (GBS), Acute disseminated encephalomyelitis (ADEM) and sensory-motor polyneuropathy (SMPN). We discuss herewith an exciting and probably first clinical case from east India, which was a diagnostic challenge and require a multispecialty approach for management. 


\section{Case History}

A 27-year-old male admitted with fever for last 18 days with a pre-admission diagnosis of Malaria. He was treated for the same but developed breathing difficulty since last four days. He was positive for SARS - CoV2 by RT-PCR. He had tachypnea with $\mathrm{SpO}_{2}$ of $92 \%$ on room air, bilateral basal crepitation and hypertension He had no associated complaints of anosmia, dysguesia or gastrointestinal symptoms. He was known diabetic. HRCT chest shows features of ARDS (Figure 1). His laboratory investigations showed leukocytosis with neutrophilia (90\%), lymphocytopenia (7\%), Neutrophil - Lymphocyte ratio of 12.86 , platelets -1.57 lacs/cmm, D - Dimer $>$ 10000, PT - $17.8 \mathrm{sec}$, S. Urea - $52 \mathrm{mg} / \mathrm{dl}$, Total Bilirubin - 7.35 $\mathrm{mg} / \mathrm{dl}$, Direct Bilirubin - $6.83 \mathrm{mg} / \mathrm{dl}$, Total protein - $6.40 \mathrm{gm} / \mathrm{dl}$, S. Albumin - $2.70 \mathrm{gm} / \mathrm{dl}$, SGOT - $72 \mathrm{U} / \mathrm{L}$, SGPT - $57 \mathrm{U} / \mathrm{L}$, S. ALP - 379 U/L, S. LDH - 624 U/L, S. C-reactive protein - 138.05, S. Ferritin - > $1000 \mu \mathrm{g} / \mathrm{dl}$, S. Lactate $-2.74 \mathrm{mmol} / \mathrm{L}$, Random blood sugar -455 $\mathrm{mg} / \mathrm{dl}$ and $\mathrm{HbA1C}-14.3 \%$. Toxicology screening was negative. He was managed and treated as per the COVID 19 management protocol of the Institute (Table 1). He also received High flow nasal oxygen therapy, shows clinical improvement and became SARS- CoV2 negative on RT-PCR after 10 days of admission.

He developed gradually progressive profound bilateral lower and right upper limb weakness and tremors within 20 days of his illness. He also developed slurring and difficulty in speech for

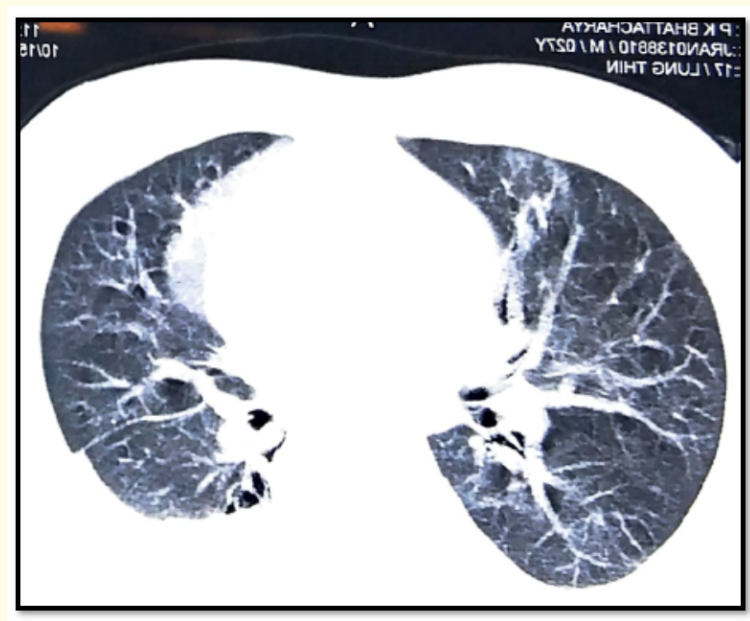

Figure 1: HRCT Chest - Bilateral lungs show ground-glass opacities, a large consolidation with air bronchogram in left lower lung lobe and thick fibrosis in the left middle lobe and inferior lingula along with left pleural effusion.

\begin{tabular}{|c|c|c|}
\hline S. No. & Name of drugs & Dosage \\
\hline 1 & Ramdesivir & $\begin{array}{c}200 \mathrm{mg} \text { stat i.v. followed by } 100 \mathrm{mg} \\
\text { i.v. OD for } 5 \text { days }\end{array}$ \\
\hline 2 & Enoxaparin sodium & 60 mg s.c. injection OD \\
\hline 3 & Regular Insulin & 18 Units i.v. infusion \\
\hline 4 & Insulin Glargine & 16 Unit s.c. after dinner \\
\hline 5 & Potassium chloride & $\begin{array}{c}1 \text { ampule in } 500 \mathrm{ml} \text { Normal saline } \\
\text { i.v. BD }\end{array}$ \\
\hline 6 & Dexamethasone & $60 \mathrm{mg}$ i.v. OD \\
\hline 7 & Meropenem & $\begin{array}{c}\text { loading dose of } 2 \text { gm followed by } 1 \\
\text { gm i.v.TDS }\end{array}$ \\
\hline 8 & VitaminC & 1 gm i.v. BD \\
\hline 9 & Tablet Ivermectin & $6 \mathrm{mg}$ BD for 7 days \\
\hline 10 & Tablet Doxycycline & $200 \mathrm{mg}$ BD for 7 days \\
\hline 11 & $\begin{array}{c}\text { Tablet } \\
\text { N-Acetyl-Cysteine }\end{array}$ & $600 \mathrm{mg}$ TDS \\
\hline 12 & Tablet Multivitamin & 1 tab OD \\
\hline
\end{tabular}

Table 1: List of drugs administered to the patient.

which neurological consultation was advised. He had Glasgow Coma Scale of $\mathrm{E}_{4} \mathrm{~V}_{2} \mathrm{M}_{6}$, reduced tone, 3/5 power in both lower limbs, absent knee and ankle jerk, plantar bilateral extensors and normal upper limb reflexes on examination. He has no sensory symptoms, neither facial or ocular muscles involvement nor bladder dysfunction. A differential diagnosis of ADEM or GBS was made and CSF examination, MRI brain, cervical and dorso-lumbar spine and Nerve conduction study (NCS) were performed to confirm the diagnosis. CSF examination shows mildly raised protein and three lymphocytes suggestive of albumino-cytological dissociation. MRI brain was normal. MRI of Dorso-lumbar spine suggests findings of transverse myelitis (Figure 2). NCS from upper and lower limbs show reduced motor nerve conduction velocities and CMAP's over both peroneals and prolonged latencies over both tibials. Sensory nerve conduction velocities were reduced over both sural nerves with prolonged latencies. F wave latencies were prolonged over-tested nerves (Table 2). NCS suggested asymmetrical sensory-motor polyneuropathy which was considered as final diagnosis and treatment with Dexamethasone and IV Ig started with which clinical improvement noted.

\section{Discussion}

SARS-CoV2 causes COVID 19 disease and most commonly presented with symptoms of ARDS, thromboembolic events or gastrointestinal dysfunctions. Recent studies report cases in which CNS involvement is significant. According to Mao L et al., more than 


\begin{tabular}{|c|c|c|c|c|c|c|c|c|c|c|}
\hline \multicolumn{11}{|c|}{ Upper limb } \\
\hline Nerve & Side & \multicolumn{2}{|c|}{ Site } & \begin{tabular}{|c|}
$\begin{array}{c}\text { Distal latency } \\
(\mathrm{mS})\end{array}$ \\
\end{tabular} & \multicolumn{2}{|c|}{ Duration (mS) } & $\operatorname{Amp}(\mathrm{mV})$ & $\begin{array}{c}\mathrm{NCV} \\
(\mathrm{m} / \mathrm{s})\end{array}$ & \multicolumn{2}{|c|}{$\begin{array}{c}\text { F wave mean latency } \\
(\mathrm{mS})\end{array}$} \\
\hline \multirow{4}{*}{$\begin{array}{l}\text { Median } \\
\text { Right }\end{array}$} & Left & \multicolumn{2}{|c|}{ Wrist } & 16.15 & \multicolumn{2}{|l|}{13.02} & 9.4 & 57.08 & & \\
\hline & \multicolumn{2}{|c|}{ Elbow } & 18.96 & \multicolumn{2}{|c|}{11.46} & 6.2 & & & & \\
\hline & \multicolumn{2}{|c|}{ Wrist } & 12.81 & \multicolumn{2}{|c|}{10.42} & 6.8 & 53.42 & & & \\
\hline & \multicolumn{2}{|c|}{ Elbow } & 17.08 & \multicolumn{2}{|c|}{10.00} & 6.9 & & & & \\
\hline \multirow{4}{*}{$\begin{array}{l}\text { Ulnar } \\
\text { right }\end{array}$} & left & \multicolumn{2}{|c|}{ Wrist } & 15.94 & \multicolumn{2}{|l|}{13.75} & 4.6 & 50.10 & & \\
\hline & \multicolumn{2}{|c|}{ Elbow } & 20.52 & \multicolumn{2}{|c|}{13.54} & 4.6 & & & & \\
\hline & \multicolumn{2}{|c|}{ Wrist } & 14.38 & \multicolumn{2}{|c|}{12.08} & 3.4 & 50.10 & \multicolumn{2}{|c|}{49.7} & \\
\hline & \multicolumn{2}{|c|}{ Elbow } & 18.65 & \multicolumn{2}{|c|}{11.56} & 2.9 & & & & \\
\hline \multicolumn{11}{|c|}{ Lower limb } \\
\hline Nerve & Side & Site & \multicolumn{2}{|c|}{ Distal latency (mS) } & Duration(mS) & \multicolumn{2}{|c|}{ Amp (mV) } & \multicolumn{2}{|c|}{$\mathrm{NCV}(\mathrm{m} / \mathrm{s})$} & $\begin{array}{l}\text { F wave mean } \\
\text { latency }(\mathrm{mS})\end{array}$ \\
\hline \multirow{4}{*}{ Peroneal } & Right & Ankle & \multicolumn{2}{|r|}{17.19} & 12.50 & & 2.2 & \multicolumn{2}{|c|}{38.63} & 49.2 \\
\hline & Knee & \multicolumn{2}{|c|}{25.42} & 11.67 & \multicolumn{3}{|c|}{1.2} & & & \\
\hline & Left & Ankle & & 16.04 & 11.56 & & 0.7 & \multicolumn{2}{|c|}{39.55} & 52.9 \\
\hline & Knee & \multicolumn{2}{|c|}{25.31} & 11.98 & & & & & & \\
\hline & Right & Ankle & & 18.96 & 12.50 & & 5.7 & & 10 & \\
\hline Tibial & Knee & & & 12.92 & & & & 46.30 & & \\
\hline IIblal & Left & Ankle & & 18.75 & 12.50 & & 5.2 & & 71 & \\
\hline & Knee & & & 12.50 & & & & & & \\
\hline
\end{tabular}

Table 2: Results of nerve conduction study (Motor) of the patient.

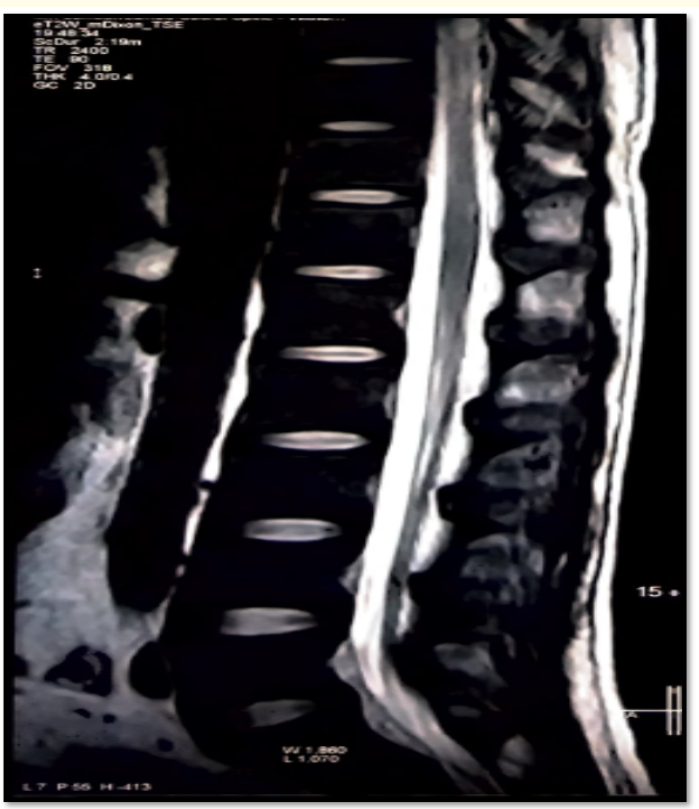

Figure 2: MRI Dorso-lumbar spine - Subtle patchy intramedullary high signal intensity on T2W seen at D 6 to D 10. one-third of patients with SARS-CoV-2 develop neurological manifestations [1]. The pathological spectrum of the disease include encephalitis, plexitis, radiculitis, encephalopathy, ischemic stroke, GBS, Multiple sclerosis, transverse myelitis and sensory-motor polyneuropathy. SARS-CoV-2 attacks the olfactory bulb and then affect the CNS through the olfactory tract in the early stages of infection [2]. Neurological impairment and demyelinating reaction appear as complications in case of severe COVID-19 [1].

Neurotropism may occur via trans-lamina cribrosa to reach the brain through the olfactory tract [3]. The SARS -CoV2 spike protein binds with host ACE 2receptor, expressed in the CNS capillary endothelium and allows the virus to penetrate the neuronal cells [4]. Budding of Virus particle lead to the onset of symptoms such as anosmia and dysguesia in the early phase of infection [1]. The pro-inflammatory state induced by the SARS-CoV2 mediated cytokine storm, and sustained neurological damage by delayed immune response may be responsible for the activation of glial cells with subsequent demyelination [5].

Zhao described a case report of GBS during SARS-CoV-2 infection [6]. GBS is an acute autoimmune fulminant demyelinating 
polyradiculopathy. It appears within 1-3 weeks of any viral infection and presented with ascending rapidly evolving flaccid motor paralysis, areflexia and bulbar muscle involvement. The sensory disturbance is mildly involved, and autonomic involvement is common. The clinical presentations in our patient are not of typical GBS, but a variant of GBS can be considered. We have limitations in not having Antiganglioside antibody test which is specific for the diagnosis of GBS. The recent studies had described less than 30 cases to date of GBS as CNS manifestation of COVID 19 disease; however, the pathophysiology of the same is still not clear.

ADEM is a syndrome of multifocal demyelination, developing weeks after an infection, presents generally with focal neurological symptoms and often with encephalopathy. There are two cases of ADEM reported to date. One have progressive dysphagia, dysarthria, and encephalopathy nine days after the onset of headache and myalgia [7]. The other presented with seizures and reduced consciousness and required intubation for respiratory failure [8]. Both patients had normal CSF and high signal intensities on MRI, typical of acute disseminated encephalomyelitis. Both of them show clinical improvement after treatment with IV Ig and steroids respectively. Symptoms of our case resemble very similar to this; hence we cannot exclude the possibility of the same.

In investigating patients with limb weakness and sensory change, it is crucial to distinguish between the disease of the peripheral nerves (e.g., GBS) and spinal cord inflammation which can present with flaccid paralysis if the anterior horn cells are involved [9]. CSF examination, neurophysiological studies, and spinal imaging are essential.

\section{Conclusion}

CNS manifestations in SARS - CoV2 infection is probably the result of direct cytolytic activity of virus itself or the indirect effect of altered hemodynamic milieu secondary to cytokine storm that result in acute CNS disorders, some of which are entirely reversible whereas few of them may progress gradually in a dormant form and reappear in future. Possibilities of psychomotor dysfunction cannot be ruled out. Hence it will be suggested that these patients must be followed up for several years at frequent intervals to assess the long term effect of the virus.

\section{Key Messages}

Sensory motor polyneuropathy is an immediate COVID 19 associated complication which will be completely reversible provided diagnosed and treated as early as possible.

\section{Acknowledgement}

No any financial support used for this study. Abbreviations used in the manuscript are mentioned below. We acknowledge the Department of Neurology, Critical care medicine, Pathology, Radiology and Laboratory Medicine for providing their support in the diagnosis of disease. We also acknowledge the effortless work of nursing staffs, technicians and physiotherapist in the management of patient and due to their effort patient is recovering very fast.

\section{Bibliography}

1. Mao L., et al. "Neurologic manifestations of hospitalized patients with coronavirus disease 2019 in Wuhan, China". JAMA Neurology 77.6 (2020): 683-690.

2. Desforges M., et al. "Human coronaviruses and other respiratory viruses: underestimated opportunistic pathogens of the central nervous system?" Viruses 12.1 (2019): 14.

3. Baig AM., et al. "Evidence of the COVID-19 virus targeting the CNS: tissue distribution, host-virus interaction, and proposed neurotropic mechanisms". ACS Chemical Neuroscience 11.7 (2020): 995-998.

4. Wrapp D., et al. "Cryo-EM structure of the 2019-nCoV spike in the prefusion conformation". Science 367.6483 (2020): 12601263.

5. Mehta P., et al. "COVID-19: consider cytokine storm syndromes and immunosuppression". Lancet 395.10229 (2020): 10331034.

6. Zhao H., et al. "Guillain-Barrè syndrome associated with SARSCoV-2 infection: causality or coincidence?" Lancet Neurology 19.5 (2020): 383-384.

7. Zhang T., et al. "COVID-19-associated acute disseminated encephalomyelitis: a case report". MedRxiv (2020).

8. Zanin L., et al. "SARS-CoV-2 can induce brain and spine demyelinating lesions". Acta Neurochirurgica (2020).

9. Solomon T and Willison H. "Infectious causes of acute flaccid paralysis". Current Opinion in Infectious Diseases 16 (2003): 375-381.

\section{Volume 4 Issue 8 August 2021}

(C) All rights are reserved by Pushpanjali R Ojha., et al. 